\title{
Use of collateral sensitivity networks to design drug cycling protocols that avoid resistance development.
}

Imamovic, Lejla; Sommer, Morten

Published in:

Science Translational Medicine

Link to article, DOI:

10.1126/scitranslmed.3006609

Publication date:

2013

Document Version

Publisher's PDF, also known as Version of record

Link back to DTU Orbit

Citation (APA):

Imamovic, L., \& Sommer, M. (2013). Use of collateral sensitivity networks to design drug cycling protocols that avoid resistance development. Science Translational Medicine, 5(204).

https://doi.org/10.1126/scitranslmed.3006609

\section{General rights}

Copyright and moral rights for the publications made accessible in the public portal are retained by the authors and/or other copyright owners and it is a condition of accessing publications that users recognise and abide by the legal requirements associated with these rights.

- Users may download and print one copy of any publication from the public portal for the purpose of private study or research.

- You may not further distribute the material or use it for any profit-making activity or commercial gain

- You may freely distribute the URL identifying the publication in the public portal

If you believe that this document breaches copyright please contact us providing details, and we will remove access to the work immediately and investigate your claim 


\section{Use of Collateral Sensitivity Networks to Design Drug Cycling Protocols That Avoid Resistance Development}

Lejla Imamovic and Morten O. A. Sommer

Sci Transl Med 5, 204ra132 (2013);

DOI: 10.1126/scitranslmed.3006609

Editor's Summary

\section{Resistance is Futile}

In an emergency situation, people are often instructed to remain calm and proceed in an orderly fashion. The same advice may apply to the current antibiotic-resistance crisis. Imamovic and Sommer now show how collateral sensitivity profiles--deciphered by treating bacteria with multiple antibiotics--can help to order drug deployment in sequences that thwart the development of antibiotic resistance.

Cells or organisms that have developed resistance to one drug sometimes display a greater sensitivity to a second drug often from a distinct structural class, a concept called collateral sensitivity. The authors tested whether application of this concept can aid in the management of bacterial infections by evolving resistance in Escherichia coli to 23 known antibiotics and then mapping the resulting collateral sensitivity and resistance profiles. On the basis of their derived collateral sensitivity network, the authors designed a new treatment framework--collateral sensitivity cycling --in which sequential treatment of $E$. coli cultures with antibiotics that display compatible collateral sensitivity profiles is predicted to select against drug-resistance development. The authors chronicled hundreds of such drug sets and validated their predictions $E$. coli and the antibiotics gentamicin and cefuroxime by showing that cyclical deployment of the drugs selected against resistance to either antibiotic. This proof of principle for collateral sensitivity cycling as a sustainable treatment regimen was then validated with two related bacterial pathogens. It remains to be seen whether deployment of cancer therapeutics in an orderly fashion also curbs drug resistance.

A complete electronic version of this article and other services, including high-resolution figures, can be found at: http://stm.sciencemag.org/content/5/204/204ra132.full.html

Supplementary Material can be found in the online version of this article at: http://stm.sciencemag.org/content/suppl/2013/09/23/5.204.204ra132.DC1.html

Related Resources for this article can be found online at: http://stm.sciencemag.org/content/scitransmed/4/140/140sr2.full.html http://stm.sciencemag.org/content/scitransmed/3/78/78ps12.full.html http://stm.sciencemag.org/content/scitransmed/5/203/203ps12.full.html http://stm.sciencemag.org/content/scitransmed/4/148/148ra116.full.html http://stm.sciencemag.org/content/scitransmed/5/192/192fs25.full.html http://stm.sciencemag.org/content/scitransmed/5/190/190ra81.full.html http://stm.sciencemag.org/content/scitransmed/5/192/192ra85.full.html http://www.sciencemag.org/content/sci/341/6153/1460.full.html http://www.sciencemag.org/content/sci/341/6153/1514.full.html

Information about obtaining reprints of this article or about obtaining permission to reproduce this article in whole or in part can be found at:

http://www.sciencemag.org/about/permissions.dtl 


\title{
ANTIBIOTICS
}

\section{Use of Collateral Sensitivity Networks to Design Drug Cycling Protocols That Avoid Resistance Development}

\begin{abstract}
Lejla Imamovic ${ }^{1}$ and Morten O. A. Sommer ${ }^{1,2 *}$
New drug deployment strategies are imperative to address the problem of drug resistance, which is limiting the management of infectious diseases and cancers. We evolved resistance in Escherichia coli toward 23 drugs used clinically for treating bacterial infections and mapped the resulting collateral sensitivity and resistance profiles, revealing a complex collateral sensitivity network. On the basis of these data, we propose a new treatment framework-collateral sensitivity cycling-in which drugs with compatible collateral sensitivity profiles are used sequentially to treat infection and select against drug resistance development. We identified hundreds of such drug sets and demonstrated that the antibiotics gentamicin and cefuroxime can be deployed cyclically such that the treatment regimen selected against resistance to either drug. We then validated our findings with related bacterial pathogens. These results provide proof of principle for collateral sensitivity cycling as a sustainable treatment paradigm that may be generally applicable to infectious diseases and cancer.
\end{abstract}

\section{INTRODUCTION}

The rapid development of drug resistance is a major threat to the successful management of infectious diseases and cancer, which collectively are responsible for more than half of disease-related deaths globally $(1,2)$. The evolution and spread of resistance reduces or entirely eliminates the utility of most drugs used for the treatment of bacterial, fungal, parasitic, and viral infections (3-6). In particular, treatment failure is rapidly increasing as a result of the alarming development of multidrug resistance in Gram-negative bacterial pathogens, such as Escherichia coli and Klebsiella, which contribute to most of the deaths caused by hospital-acquired infections (7). The burden of resistance is also evident with cancer therapy, in which drug resistance remains a major cause of treatment failure and death among cancer patients $(8,9)$.

Despite the desperate need for new infectious disease therapies, limited progress has been made in the discovery of first-in-class antibacterial drugs to balance the relentless resistance development $(10,11)$. Another promising approach for addressing drug resistance is through rational treatment (deployment) strategies. The most prominent strategies are drug combination therapies and drug stewardship programs.

Combination therapies have been deployed clinically both to prevent the rise of resistance and to improve treatment efficacy. The ability of drug combinations to improve treatment outcomes has been demonstrated in a variety of disease areas, including HIV infection $(4,12-15)$. It has also been demonstrated in bacteria that antagonistic drug combinations, in which the constituent drugs counteract each other's effects, can be used to select against resistance that results from suppressive drug interactions (16).

Antimicrobial stewardship programs aim to decrease drug resistance by avoiding unnecessary use and choosing an adequate dose with the shortest effective duration of treatment $(17,18)$. Drug cycling programs are a form of antimicrobial stewardship that involves the scheduled rotation of distinct antibiotic classes that exhibit comparable antimicrobial activity spectra. The rotation can include any number of drugs, but must be concluded with reapplication of the original drug. The current rationale behind antibiotic cycling relies on the fact

'Department of Systems Biology, Technical University of Denmark, DK-2800 Lyngby, Denmark. ${ }^{2}$ Novo Nordisk Foundation Center for Biosustainability, Technical University of Denmark, DK-2970 Hørsholm, Denmark.

${ }^{*}$ Corresponding author. E-mail: msom@bio.dtu.dk that evolution of drug resistance is related to drug exposure; thus, by withdrawing a drug from the clinic for a given period, the selective benefit of resistance is removed, resulting in a decrease in resistance frequencies (19-21). These predicted advantages of conventional antibiotic cycling rest on the assumption that resistance is accompanied by a biological fitness cost. The larger the fitness cost, the more rapidly the resistance frequency of a population will decrease in the absence of the selective pressure imposed by drug treatment (22). Thus, if drug use is reduced, the resistant mutants with the highest fitness costs are expected to be outcompeted by their nonresistant counterparts and disappear from the bacterial population.

However, the spread of resistant bacterial strains still occurs even though resistance can be costly in terms of bacterial fitness (23-25). In addition, not all resistance carries fitness cost $(26,27)$, and the fitness cost can be ameliorated by compensatory mutations $(22,28)$. Finally, it has been shown that resistant subpopulations of bacteria can persist for decades after drug removal (29).

Current antibiotic cycling strategies implement cycles of drug restriction and prioritization on an ad hoc basis, and the benefits of this strategy remain controversial (30-33). Here, we propose a fundamentally different approach to drug cycling that builds on the finding that the development of resistance to one drug can perturb drug susceptibility profiles in the bacterial cell; this adaptation results in collateral resistance and collateral sensitivity toward other drugs through perturbation of gene expression networks that affect a cell's vulnerability to chemicals (34-36) (Fig. 1, A to C). The collateral effects of resistance evolution provide a rationale for a new treatment strategy: collateral sensitivity cycling (Fig. 1D).

Consider a target bacterial population undergoing treatment with drug A (Fig. 1D). When resistance to drug A evolves within the target population, treatment can be switched by deployment of drug B, to which the drug A-resistant population displays collateral sensitivity (Fig. 1D). With this treatment regimen, the drug A-resistant bacterial subpopulation would be eradicated more effectively than the subpopulation that has not developed resistance to drug A. If the drugs are chosen carefully to have reciprocal collateral sensitivity profiles, collateral sensitivity cycling should limit evolution of drug resistance in the target bacterial population. Because collateral sensitivity derives from changes 
in cellular networks that drive the evolution of drug resistance, this phenomenon is a systems-level characteristic and has been observed in diverse bacteria $(34,37)$, viruses $(38)$, and cancers $(9,39)$. Thus, if collateral sensitivity can be exploited to counter resistance and maintain drug effectiveness in one biological system, it should be generally deployable within many different disease areas in which evolution of drug resistance occurs. We hypothesized that it would be possible to identify sets of drugs with reciprocal collateral sensitivity profiles that enable their application in a cyclical manner that kills bacteria while sustainably selecting against drug resistance (Fig. 1D). Because Gramnegative bacteria are associated with prolonged hospitalization, treatment failure, and increased mortality rates worldwide $(7,40-42)$, we tested the concept of collateral sensitivity cycling in the Gram-negative bacterium E. coli.

\section{RESULTS}

\section{Collateral sensitivity profiles}

We evolved parallel lineages of E. coli (Materials and Methods) to become resistant to 23 clinically relevant antibiotics spanning 11 distinct
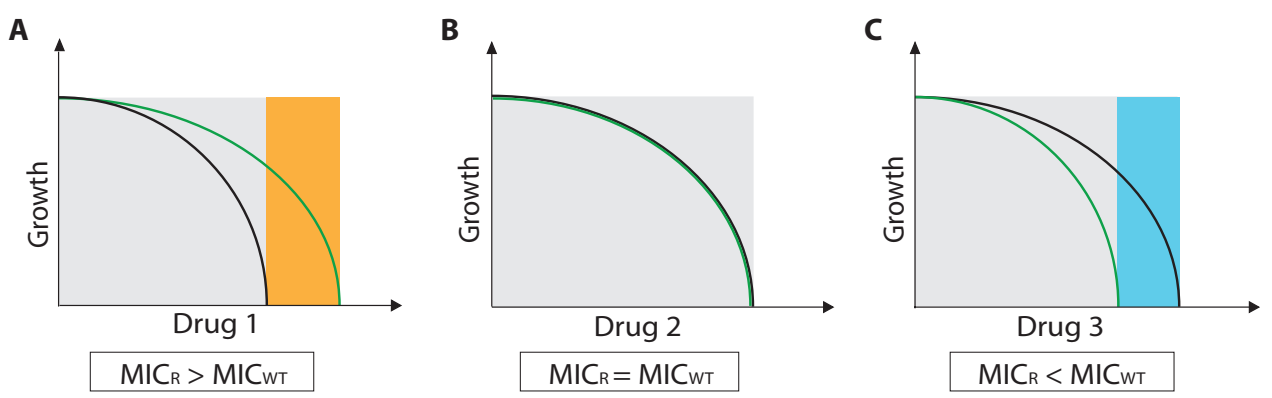

Fig. 1. Collateral sensitivity and its application in drug $D$ cycling. (A to $\mathbf{C}$ ) Determination of drug susceptibility profiles is based on the growth of a WT (black line) and resistant strain (green line) as a function of varying drug concentrations. (A) Collateral resistance. As the concentration of drug 1 increases, a resistant strain with collateral resistance to this drug will outcompete the WT (orange shade). (B) No change in susceptibility. As

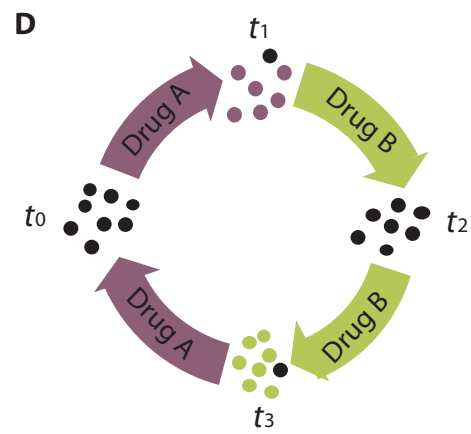

chemical classes and mechanisms of action (Table 1 and table S2). For each resistant isolate, we performed dose-response experiments to determine the susceptibility profiles (Fig. 1, A to C) for the 23 antibiotics (fig. S1). The resistance levels acquired during adaptation for 23 E. coli strains as measured by minimum inhibitory concentrations (MICs) in culture were equal to or above the current clinical breakpoints according to The European Committee on Antimicrobial Susceptibility Testing (EUCAST) (table S1). Seventeen of the 23 resistant E. coli isolates exhibited collateral sensitivity toward at least one other drug (Fig. 2). With the exception of the cefuroxime-resistant strain, which showed no collateral resistance toward the $\beta$-lactam amoxicillin, all resistant strains were collaterally resistant toward drugs from the same chemical class and to at least one drug from a different class (Fig. 2 and fig. S1). Excluding drugs with the same mechanism of action, we observed collateral resistance up to a $>32$-fold increase in the MIC compared to the wildtype E. coli (WT). A high level of collateral resistance was observed between amphenicol and tetracycline drug classes as well as between amphenicol, $\beta$-lactam, and quinolone drug classes (Fig. 2A and fig. S1). The $\beta$-lactam and quinolone drug classes are commonly used in clinical cycling programs $(20,33)$, and the collateral resistance observed could contribute to the failure of such drug cycling programs.

Although we observed collateral sensitivity with magnitudes of down to an eightfold reduction of MIC, the most frequently observed collateral sensitivity was a twofold reduction in MIC relative to WT. E. coli that acquired resistance to the aminoglycoside chemical class, which is frequently used for treatment of hospital-acquired infections with multidrug-resistant Gram-negative pathogens (42) (details on drug common use are listed in table S2), exhibited increased sensitivity to 14 unrelated drugs from several chemically diverse drug classes, including $\beta$-lactams, polymyxins, macrolides, tetracyclines, chloramphenicol, fosfomycin, and nitrofurantoin (Fig. 2). The collateral sensitivity of isolates resistant to different drugs within a particular chemical class was not always uniform. For instance, collateral sensitivity toward polymyxins, fosfomycin, and azithromycin was observed for E. coli strains resistant to the aminoglycosides amikacin, gentamicin, and kanamycin, but not for E. coli strains resistant to the aminoglycoside streptomycin. On the other hand, an isolate resistant to the amphenicol chemical class (chloramphenicol) increased sensitivity toward streptomycin but not toward any other aminoglycosides tested (Fig. 2). These findings indicate that drugs belonging to a specific class do not always induce the same collateral sensitivity. Instead, drug-specific resistance evolution plays a role in determining the collateral sensitivity profiles. 
Table 1. List of antibiotics used in the study.

\begin{tabular}{|c|c|c|c|}
\hline Antibiotic & Abbreviation & Class & Target \\
\hline Amikacin & AMI & Aminoglycoside & Protein synthesis, $30 \mathrm{~S}$ \\
\hline Gentamicin & GEN & Aminoglycoside & Protein synthesis, $30 \mathrm{~S}$ \\
\hline Kanamycin & KAN & Aminoglycoside & Protein synthesis, $30 \mathrm{~S}$ \\
\hline Streptomycin & STR & Aminoglycoside & Protein synthesis, $30 \mathrm{~S}$ \\
\hline Ampicillin & AMP & $\beta$-Lactam & Cell wall \\
\hline Amoxicillin & AMX & $\beta$-Lactam & Cell wall \\
\hline Piperacillin & PIP & $\beta$-Lactam & Cell wall \\
\hline Cefuroxime & CFX & $\beta$-Lactam & Cell wall \\
\hline Cefepime & CFP & $\beta$-Lactam & Cell wall \\
\hline Nalidixic acid & NAL & Quinolone & DNA gyrase \\
\hline Ciprofloxacin & CIP & Quinolone & DNA gyrase \\
\hline Levofloxacin & LEV & Quinolone & DNA gyrase \\
\hline Tetracycline & TET & Tetracycline & Protein synthesis, 305 \\
\hline Minocycline & MIN & Tetracycline & Protein synthesis, 305 \\
\hline Tigecycline & TGC & Tetracycline & Protein synthesis, 305 \\
\hline Chloramphenicol & $\mathrm{CHL}$ & Amphenicol & Protein synthesis, $50 \mathrm{~S}$ \\
\hline Azithromycin & $A Z Y$ & Macrolide & Protein synthesis, $50 \mathrm{~S}$ \\
\hline Colistin & $\mathrm{COL}$ & Polymyxin & Lipopolysaccharide \\
\hline Polymyxin B & POL & Polymyxin & Lipopolysaccharide \\
\hline Fosfomycin & FOS & Fosfomycin & Cell wall biogenesis \\
\hline Rifampicin & RIF & Rifamycin & RNA polymerase \\
\hline Nitrofurantoin & NIT & Nitrofuran & Multiple \\
\hline Trimethoprim & TRI & Dihydrofolate reductase inhibitor & Folic acid biosynthesis \\
\hline
\end{tabular}

\section{Identification of collateral sensitivity cycles}

Given that the evolution of resistance toward 23 clinically relevant antibiotics led to substantial collateral sensitivity across nine diverse chemical classes, we identified all possible collateral sensitivity cycles within our data set. First, we created a collateral sensitivity network for E. coli wherein the nodes of the network are the drugs and the directional edges of the network represent collateral sensitivities resulting from resistance evolution toward each of these drugs (Fig. 3A). For example, when a strain develops resistance to gentamicin, several drugs can be chosen to which gentamicin-resistant strains will be collaterally sensitive (for example, colistin, nalidixic acid, or cefuroxime) (Fig. 3A). Again, when resistance evolves to the next drug (for example, colistin), the treatment can be switched to the next possible option to which the colistin-resistant strain will be collaterally sensitive and so on.

We analyzed all possible sequential drug deployment strategies composed of two to four drugs (Fig. 3A) that formed closed loops (cycles) within our collateral sensitivity network. We identified 207 possible cycles (Fig. 3B and table S3), suggesting that several options exist for deployment of tailored collateral sensitivity cycles in response to specific resistance patterns. Certain drugs were included more frequently in the identified collateral sensitivity cycles. For example, of the 22 two-drug cycles identified (Fig. 3C), 59\% involved drugs belonging to the aminoglycoside, polymyxin, or tetracycline chemical classes as a result of the strong reciprocal collateral sensitivity profile observed between those drug groups (Figs. $2 \mathrm{~A}$ and $3 \mathrm{C}$ and table S3).

However, not all drugs can be used in simple two-drug cycles. For instance, sequential deployment of drugs with collateral resistance will provide a selective advantage to the resistant strain over the WT and lead to amplification of resistance. Yet, drugs with collateral resistance profiles can be included in the same collateral sensitivity cycle when interspersed by a drug toward which the drugs have a collateral sensitivity. In such cases, collateral sensitivity cycling with two different aminoglycoside drugs with collateral resistance can be achieved if quinolone and $\beta$-lactam drugs are included in the cycle (for example, cefuroxime $\rightarrow$ gentamicin $\rightarrow$ nalidixic acid $\rightarrow$ amikacin) (Fig. 3A and table S3). Sequential deployment of such drug sets based on their collateral sensitivity profiles should continue to select against resistance despite collateral resistance between some of the cycle component drugs.

\section{Selection against drug resistance through collateral sensitivity cycling}

To test whether collateral sensitivity cycling of drugs can suppress WT resistance development over time, we chose two clinically relevant drugs from the aminoglycoside and $\beta$-lactam classes (gentamicin and cefuroxime) that exhibited reciprocal collateral sensitivity profiles (Fig. 2A). First, we created isogenic indicator E. coli strains that express either cyan fluorescent protein (CFP) or yellow fluorescent protein 
A

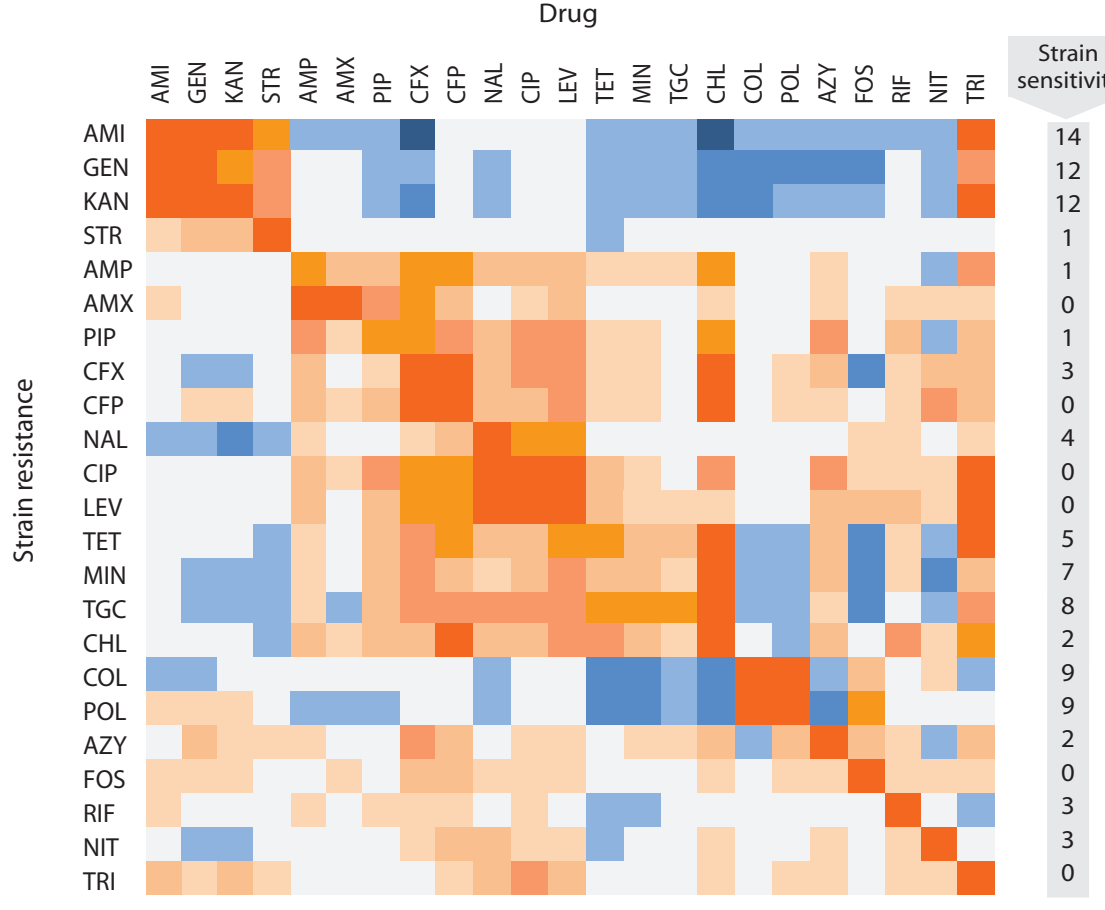

Drug sensitivity
B

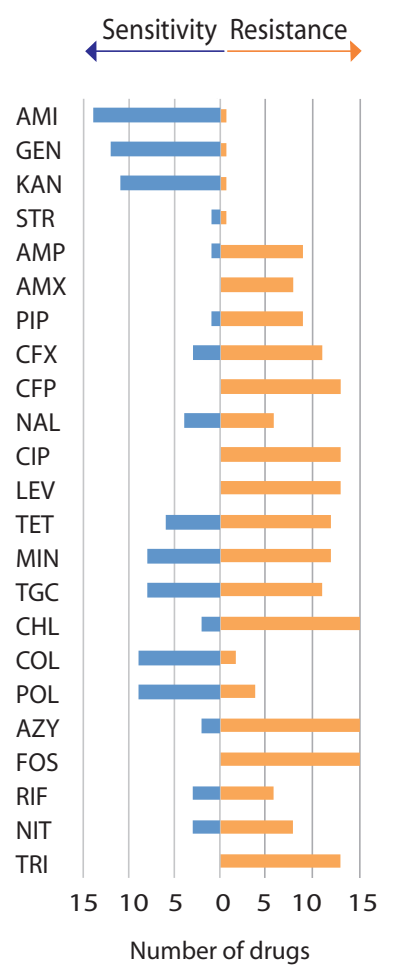

Number of drugs

$$
\begin{array}{llllllllllll}
32 \mathrm{x} & 16 \mathrm{x} & 8 \mathrm{x} & 4 \mathrm{x} & 2 \mathrm{x} & 0 & 2 \mathrm{x} & 4 \mathrm{x} & 8 \mathrm{x} & 16 \mathrm{x} & 32 \mathrm{x} \\
\hline & \text { Sensitivity } & \text { MICWT } & \text { Resistance } \\
\hline
\end{array}
$$

Fig. 2. Collateral sensitivity profiles of drug-resistant $E$. coli strains. (A) A heat map showing drug susceptibility profiles of drug-resistant $E$. coli strains relative to the WT. Susceptibility profiles were defined on the basis of MIC inhibition curves (fig. S1). (B) Distribution of drug collateral sensitivity and collateral resistance for 23 drug-resistant $E$. coli strains (drugs from the same class are excluded). Throughout the figure, blue coloring refers to collateral sensitivity, orange coloring refers to collateral resistance, and white coloring refers to no change in susceptibility relative to WT. Drug abbreviations are shown in Table 1.
(YFP) (Materials and Methods). Then, we used the CFP-labeled E. coli strain to select resistant mutants that grew in gentamicin at 32 times the MIC of the WT strain $\left(32 \times \mathrm{MIC}_{\mathrm{WT}}\right)$ by repeated exposures to increasing concentrations of gentamicin. Next, we mixed the YFP-labeled WT strain and the CFP-labeled gentamicin-resistant strain and exposed this mixed population to a wide range of concentrations of cefuroxime. From these experiments, we discovered that gentamicin resistance can be selected against at cefuroxime concentrations of $4 \mu \mathrm{g} / \mathrm{ml}$ (Fig. 4A). Through a similar approach, we found that gentamicin concentrations of $1 \mu \mathrm{g} / \mathrm{ml}$ select against cefuroxime resistance (Fig. 4B).

We then conducted an experiment to provide proof of principle for collateral sensitivity cycling. We gradually evolved a CFP-labeled E. coli WT population to become resistant toward gentamicin during 8 days of repeated exposure. Once $32 \times \mathrm{MIC}_{\mathrm{WT}}$ resistance had been achieved, the CFP-labeled gentamicin-resistant cells were mixed 1:1 with YFP-labeled WT cells, and the mixed population was exposed to cefuroxime $(4 \mu \mathrm{g} / \mathrm{ml})$ overnight. This treatment switch led to the preferential and complete killing of the CFP-labeled gentamicin-resistant cells (Fig. 4C). Then, we evolved the surviving YFP-labeled cells to become resistant to cefuroxime during 8 days of repeated exposure. Once $128 \times \mathrm{MIC}_{\mathrm{WT}}$ resistance had been achieved, the YFP-labeled cefuroxime-resistant cells were mixed with CFP-labeled WT cells and exposed to gentamicin $(1 \mu \mathrm{g} / \mathrm{ml})$, resulting in the preferential and complete killing of the YFP-labeled cefuroxime-resistant cells and survival of the CFP-labeled WT cells (Fig. 4C). These data demonstrated how collateral sensitivity cycling can be applied to select against resistance and maintain WT resistance levels to clinically relevant drugs. Note that collateral sensitivity and not the fitness cost of resistance is the basis of the selection against resistance, because we readily recovered resistant mutants from the mixed population in the absence of drug selection (Fig. 4, A and B).

To assess the reproducibility of collateral sensitivity phenotypes resulting from resistance development, we repeated the competition assay by changing the drug toward which each of the labeled strains was adapted. Again, we obtained comparable results, which is consistent with the assumption that evolutionary pathways leading to collateral sensitivity have a repeatable adaptive basis (fig. S2).

\section{Effect of collateral sensitivity on killing kinetics and mutant selection}

We have shown that sequential dosing of gentamicin and cefuroxime constitutes a collateral sensitivity cycle that selects against resistance. However, in clinical settings, all infecting pathogens must be erad- 
A

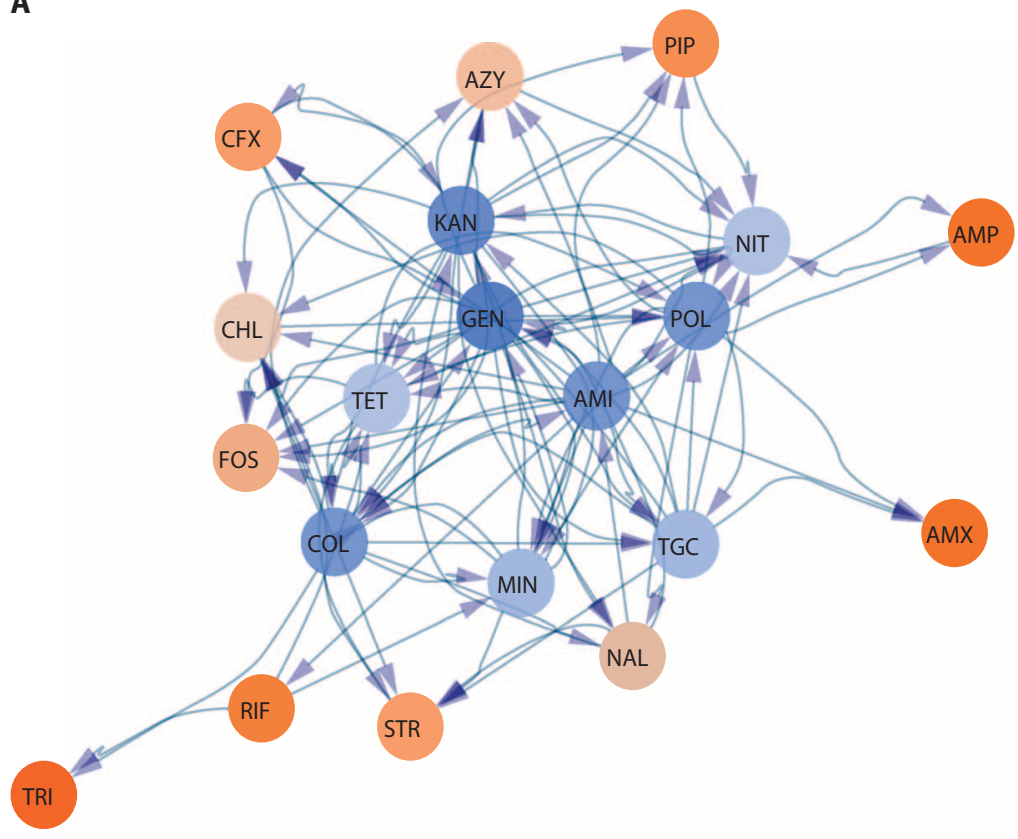

B

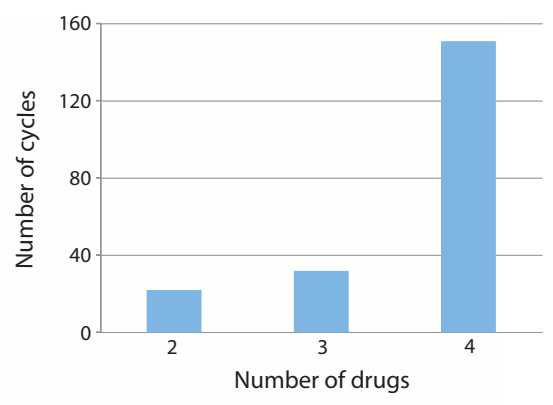

C

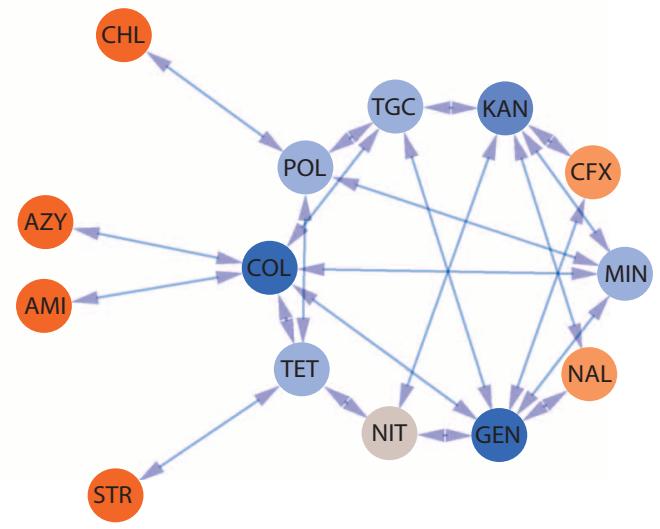

Fig. 3. Collateral sensitivity cycles. (A) Collateral sensitivity network of $E$. coli. Nodes of the network are the drugs, and the edges of the network represent collateral sensitivities resulting from resistance acquired to those drugs (Table 1). For example, if E. coli has evolved resistance to rifampicin (RIF), it displays collateral sensitivity to tetracycline (TET), minocycline (MIN), tigecycline (TGC), and trimethoprim (TRI). The color coding of the nodes (orange to blue) is proportional to the num- ber of edges proceeding from a particular node and represents the number of drugs with collateral sensitivity. (B) Distribution of the number of potential collateral sensitivity cycles as a function of the number of drugs included in the cycle. (C) Drug pairs with reciprocal collateral sensitivity. The color coding of the nodes (orange to blue) is proportional to the number of collateral sensitivity pairs of which a given drug is a part (Table 1). icated; hence, drug concentrations above $\mathrm{MIC}_{\mathrm{WT}}$ must be applied. We tested whether collateral sensitivity affected the kill kinetics of the resistant bacteria when drugs were applied at concentrations that exceeded the $\mathrm{MIC}_{\mathrm{WT}}$. We determined time-kill curves for WT E. coli and strains resistant to cefuroxime or gentamicin in the presence of $16 \times \mathrm{MIC}_{\mathrm{WT}}$ concentrations of either drug. In both cases, resistant strains were eradicated more quickly than the WT when exposed to the drug to which they were collaterally sensitive (Fig. 5, A and B). This finding has important implications for future clinical application, because it suggests that treatment within the collateral sensitivity cycling paradigm could offer more effective eradication of resistant infections compared to cycling of drugs without reciprocal collateral sensitivities.

We have characterized collateral sensitivity in terms of the drug concentration, which inhibits measurable growth of WT and resistant strains defined by their MIC. However, drug resistance can be selected for at concentrations exceeding the MIC during therapy if the drug concentration falls below the mutant prevention concentration (MPC). The MPC is the lowest concentration of a drug that prevents resistant mutants from appearing in a population of a specified size $(43,44)$. To prevent resistance development within the target population, drug concentrations at the site of infection should be above the MPC. We speculated that the MPC would be reduced proportionally to the reduction in MIC when collateral sensitivity is observed. This would provide further advantage of collateral sensitivity cycling in a clinical setting. To test this hypothesis, we determined the MPC of gentamicin and cefuroxime for WT E. coli and for strains resistant to gentamicin and cefuroxime. We found that both gentamicin- and cefuroximeresistant strains had lower MPC values relative to WT for the drugs to which they were collaterally sensitive (Fig. 5C). Hence, the administration of drugs based on collateral sensitivity can prevent the appearance of resistant mutants at a lower concentration for strains with resistance compared to the WT. This further supports the hypothesis that collat- 
A

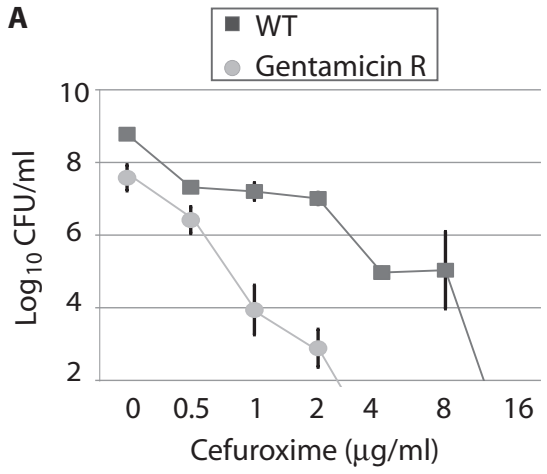

B

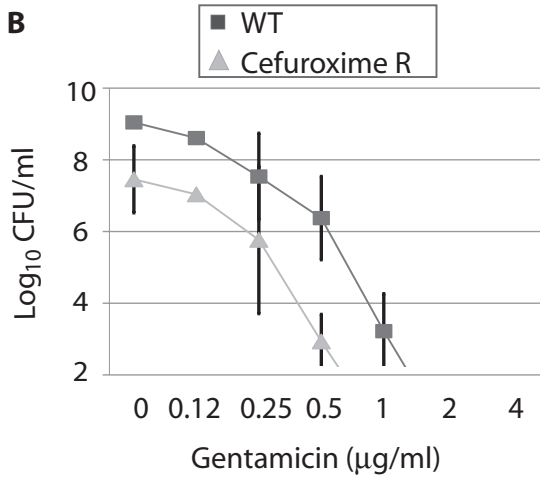

C
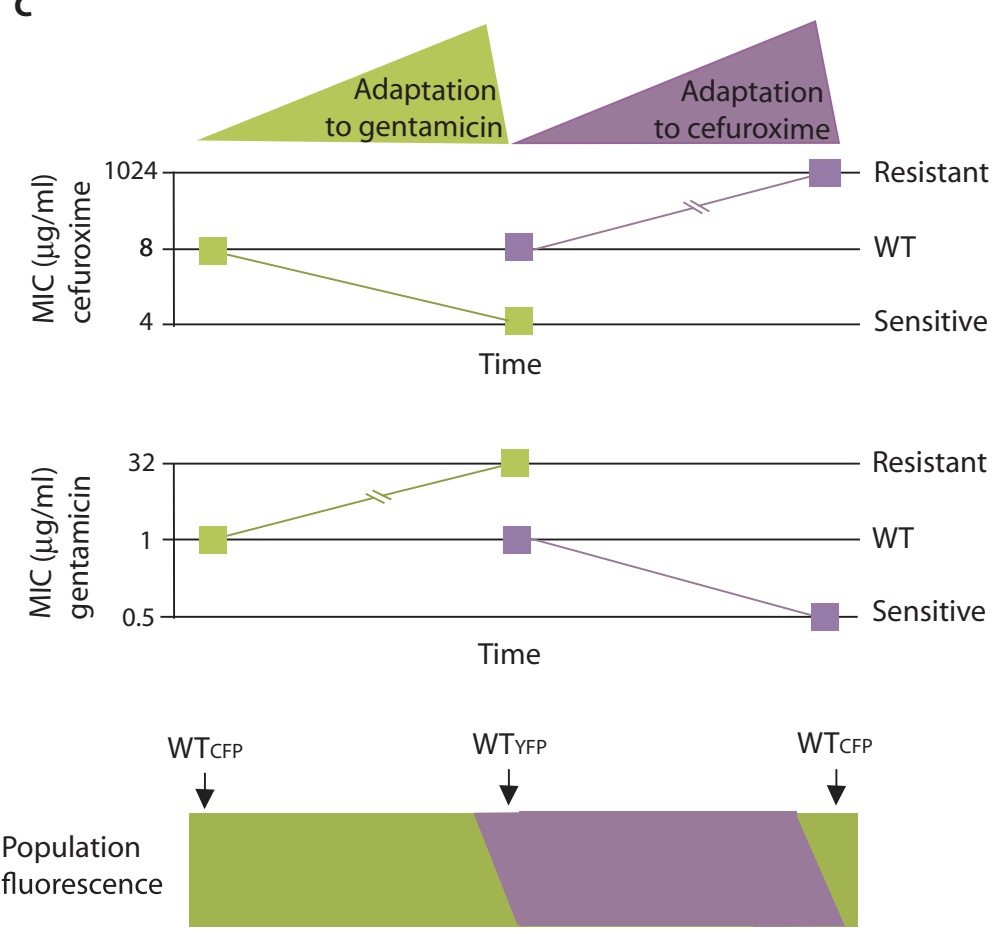

$\begin{array}{lllllllllllllllll}1 & 2 & 3 & 4 & 5 & 6 & 7 & 8 & 9 & 10 & 11 & 12 & 13 & 14 & 15 & 16 & 17\end{array}$

Time (days)

plete eradication of the CFP-labeled population. The surviving YFP-labeled population was then exposed to cefuroxime over an 8-day period until a 128 -fold increase in $M_{\text {IC }}$ was achieved. At this point, the YFPlabeled cefuroxime-resistant population was mixed with a CFP-labeled WT population and exposed to gentamicin $(1 \mu \mathrm{g} / \mathrm{ml})$, resulting in the eradication of the YFP-labeled cefuroxime-resistant population. The data were consistent across three replicates. Green color refers to treatment with gentamicin, and violet refers to treatment with cefuroxime. In the bottom panel, the proportion of the population that is CFP-labeled (green) or YFP-labeled (violet) is represented during the 17-day drug exposure period. eral sensitivity cycling can reduce the likelihood of multidrug resistance evolution, because lower drug concentrations are needed to prevent the selection of multidrug-resistant strains compared to cycling of drugs without reciprocal collateral sensitivity profiles.

\section{Collateral sensitivity in clinical isolates of $E$. coli}

To explore the clinical relevance of collateral sensitivity networks, we determined the collateral sensitivity profiles of two pathogenic E. coli isolates (Materials and Methods). The pathogenic isolates were adapted to eight antibiotics that spanned various chemical classes. The eight antibiotics were chosen as a representative subset of the original 23 antibiotics (Table 1), and the distributions of collateral resistance and sensitivity observed for the strains with resistance toward both drug sets were similar (fig. S3). We determined the collateral effects of drug resistance development for each isolate and found that all resistant isolates exhibited collateral sensitivity (Fig. 6). For one of the clinical isolates, we even observed collateral sen- sitivity with magnitudes of down to 32-fold compared to $\mathrm{MIC}_{\mathrm{WT}}$ (figs. S4 and S5). The susceptibility profiles of the evolved clinical E. coli isolates agreed well, in general, with those of the laboratory strain (Fig. 6). Indeed, $94 \%$ of the conditions tested showed either the same or no change in their response to drug exposure after resistance evolution. Six percent (four conditions) of the resistance-induced collateral susceptibility changes differed between the laboratory strain and the two clinical isolates. In three of four cases, the differences observed in collateral sensitivity and collateral resistance between the isolates involved strains resistant to nitrofurantoin or changes in the susceptibility toward nitrofurantoin. Nitrofurantoin is used in the treatment of complicated urinary tract infection (45) (table S2), and it affects several targets in the bacterial cell (46), which may, at least partially, explain the variation observed with respect to collateral sensitivity and collateral resistance for the strains tested. This similarity suggests that collateral sensitivity profiles are largely preserved across multiple isolates. Overall, the robustness of the collateral sensitivity profiles for the evolved 
A

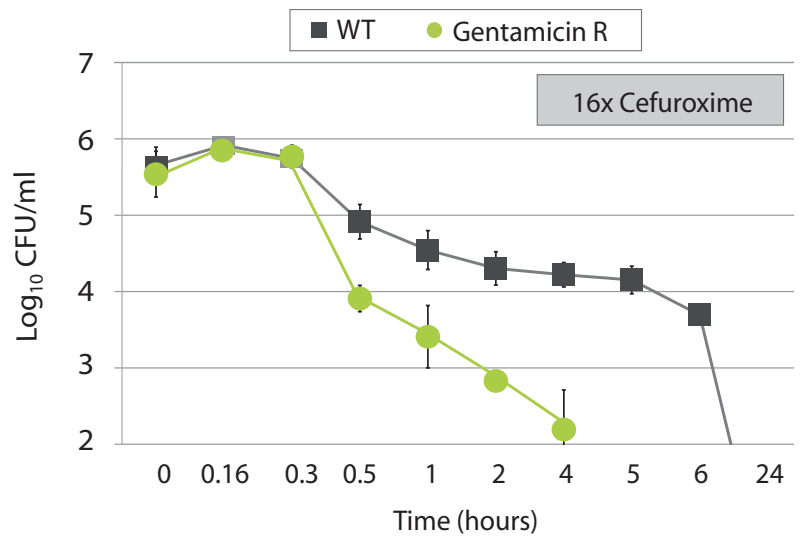

B

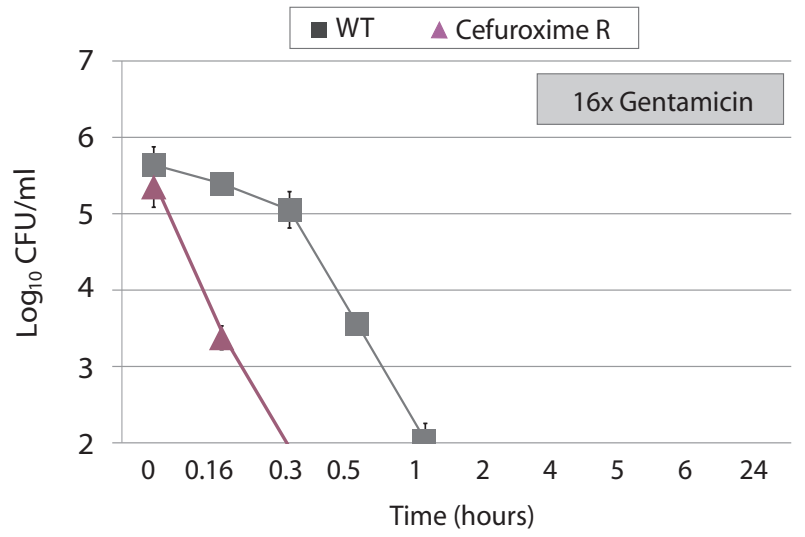

C
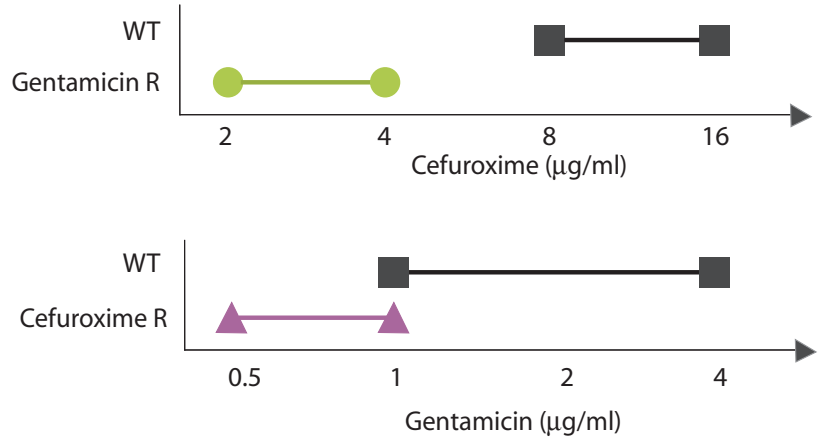

Fig. 5. Alteration of the killing dynamic and mutant selection window of resistant $E$. coli strains by collateral sensitivity. (A and B) In the presence of a drug to which resistant strains are collaterally sensitive, both gentamicin- and cefuroxime-resistant strains were eradicated faster than the WT. Results are means of three replicates \pm SD. (C) Strains resistant to gentamicin or cefuroxime also had a lower MPC relative to WT when exposed to drugs to which they were collaterally sensitive.

clinical isolates suggests that general collateral sensitivity cycles could be effective for treating a wide range of $E$. coli pathogens.

\section{DISCUSSION}

This study provides proof of principle that an underappreciated side effect of resistance, collateral sensitivity, can be used to target drug

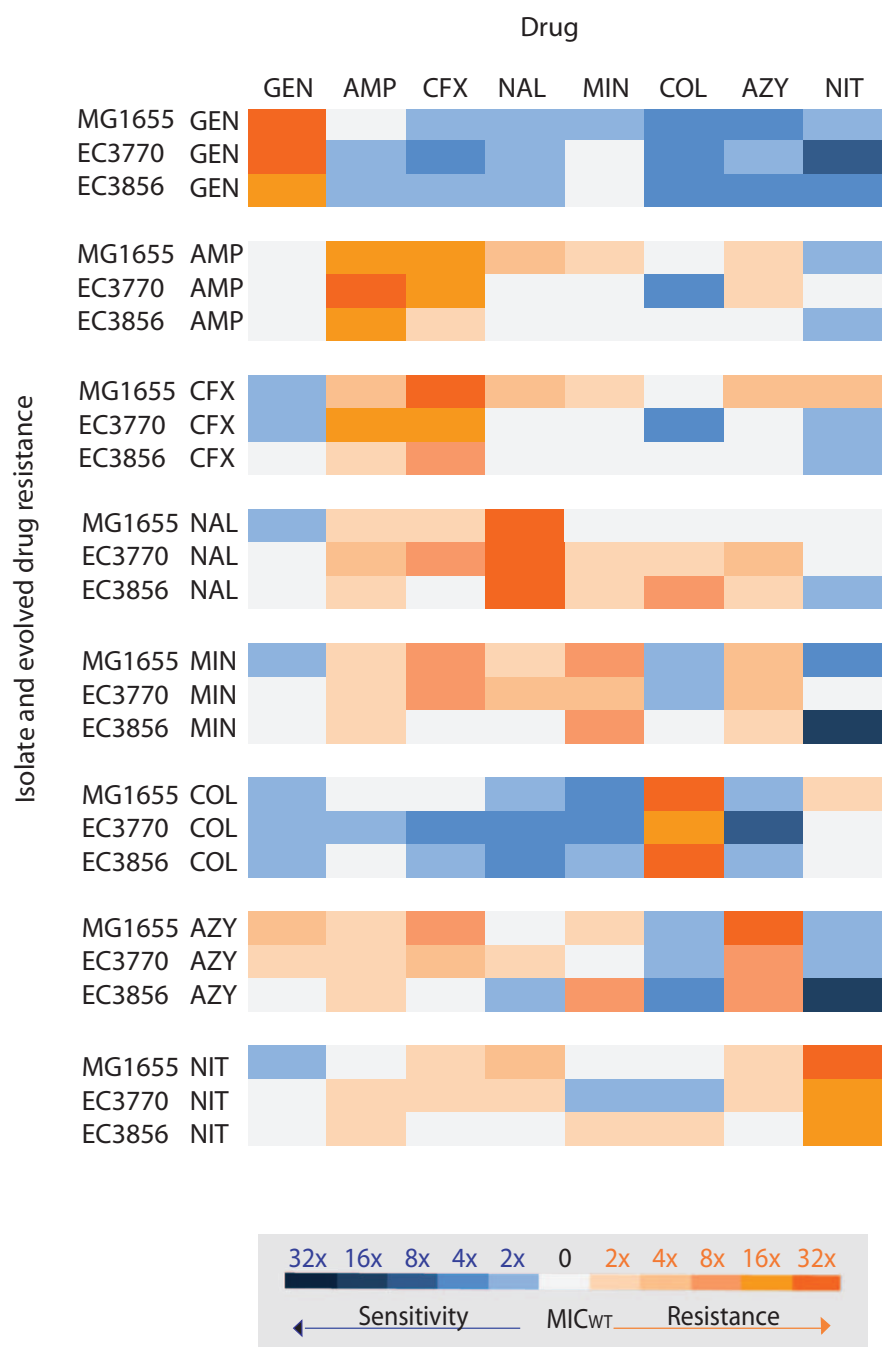

Fig. 6. Collateral sensitivity profiles for $E$. coli clinical isolates. A heat map showing drug susceptibility profiles of two resistant $E$. coli pathogenic isolates (EC3770 and EC3856) and the MG1655 strain, which evolved resistance to eight drugs. Susceptibility profiles were defined on the basis of MIC inhibition curves for the resistant strains and WT (figs. S1, S4, and S5). Blue coloring refers to collateral sensitivity, orange coloring refers to collateral resistance, and white coloring refers to no change in susceptibility relative to WT. Drug abbreviations are listed in Table 1.

resistance when selected drugs are cycled optimally. In collateral sensitivity cycling, selection against resistance was based primarily on an increase in drug sensitivity imposed by rationally selected drugs with reciprocal collateral sensitivities, which is fundamentally different from the principle of current drug cycling, which relies on the dilution of resistant strains in the absence of selection for resistance and resulting from fitness costs of resistance.

The aim of any drug treatment is the complete eradication of the disease by exposing disease-causing cells to therapeutic concentrations of drug within the host. In principle, drug treatment based on collateral sensitivity cycling should exhibit superior efficacy in patients infected with both the fully susceptible bacterial population and resistant mutants compared to cycling of drugs without compatible collateral sensitivity profiles. 
Our findings suggest that collateral sensitivity cycling should be applicable for the clinical management of Gram-negative bacterial infections using already approved antibiotics. It is, perhaps, even more important to apply this principle for treatment of chronic infections and infection in which multiple pathogens are involved, such as cystic fibrosis (47). In such cases, the application of three- or four-drug cycles could enhance the efficacy of treatment when designed optimally on the basis of common pathogen susceptibility profiles. However, further drug resistance and sensitivity programs are needed to explore the concept within these therapeutic areas, and clinical studies are needed to test whether our expectations are confirmed in hospital settings.

Here, we demonstrated the principle of collateral sensitivity cycling for resistance acquired through adaptive evolution, which is the main pathway leading to resistance in infectious diseases, such as tuberculosis and malaria, and in cancer. Indeed, drug switching based on collateral sensitivity observed in cancer treatment has shown successful therapeutic results $(48,49)$. We anticipate that collateral sensitivity cycling will contribute to the sustainable use of drugs in the clinic for the management of diseases in which evolutionary drug resistance is a concern.

\section{MATERIALS AND METHODS}

\section{Media, bacterial strains, and drugs}

Resistant strains were derived from E. coli MG1655 and two pathogenic E. coli strains EC3770 and EC3856 provided by the laboratory of clinical microbiology at Hvidovre Hospital (Denmark). All strains used in the study are derived from E. coli MG1655. Strains were adapted to the 23 antibiotics listed in Table 1 . All experiments were conducted in modified LB medium [peptone (10 g/liter), yeast extract (5 g/liter), and $\mathrm{NaCl}$ (10 g/liter)]. All drug solutions were prepared from powder stock and filter-sterilized. The drug stocks were stored at $-20^{\circ} \mathrm{C}$ for maximum of 90 days.

\section{Strain adaptation}

Strains evolved resistance to selected drugs by gradient plate method (34) on LB agar. The selection for resistance was repeated during a period of 10 days. Overnight cultures of $E$. coli were streaked onto the whole gradient plate. After 18 hours of incubation at $37^{\circ} \mathrm{C}$, the edge of growth was swept with a loop and applied to a fresh gradient plate. The process was repeated until growth was obtained from the whole plate. After this procedure, strains evolved resistance to 21 drugs (Table 1 and table S1). Strains adapted to polymyxin drug class (colistin and polymyxin B) with gradient plates did not correlate between the resistance on solid and liquid medium (strain resistance in liquid medium was fourfold lower than the level of adaptation on gradient plate). Therefore, the resistance to colistin and polymyxin B was evolved by serial passage in liquid medium according to the following protocol. About $10^{6}$ cells from overnight cultures were added to twofold serial dilution of colistin or polymyxin B in $10 \mathrm{ml}$ of LB broth. After the overnight incubation at $37^{\circ} \mathrm{C}$, absorbance measurements at $600 \mathrm{~nm}\left(A_{600}\right)$ were taken with a plate reader (Epoch BioTek). The cultures grown at the highest antibiotic concentration for which $A_{600}$ value was above 0.4 were used for further adaptation. Again, about $10^{6}$ cells from overnight cultures were added to twofold serial dilution of colistin or polymyxin B and incubated overnight. This step was repeated for 10 days. All strains were stored in $15 \%$ glycerol at $-80^{\circ} \mathrm{C}$.

\section{MIC and $\mathrm{IC}_{90}$ determination}

Strains were taken from $-80^{\circ} \mathrm{C}$, streaked onto $\mathrm{LB}$ agar supplemented with antibiotic (Table 1), and incubated overnight at $37^{\circ} \mathrm{C}$. For MIC determination, overnight cultures were prepared from cultures from LB agar plates. About $1 \times 10^{4}$ cells were prepared form overnight cultures and grown at $37^{\circ} \mathrm{C}$ with shaking in 96-well microtiter plates containing $200 \mu \mathrm{l}$ of medium per well. MICs were determined in logarithmic drug concentration gradient with twofold dilutions. Endpoint absorbance measurements $\left(A_{600}\right)$ were taken after 18 hours of incubation with a plate reader (Epoch Biotech) and background-subtracted. The percentage of inhibition was calculated according to the following formula: $1-\left[A_{600} \mathrm{drug} / A_{600}\right.$ control]. The inhibitory concentration was defined as the lowest concentration of the drug that inhibited $90 \%$ of the growth of the strain tested $\left(\mathrm{IC}_{90}\right)$. For strains in which inhibition was not uniform across the three sets of experiments performed, two additional sets of experiments were performed to determine drug susceptibility. The results obtained are summarized in Fig. 2 .

\section{Susceptibility profiles}

Strain susceptibility profile was determined relative to the WT (Fig. 2 and fig. S1). Collateral resistance was defined when the growth of a resistant strain was inhibited at a higher drug concentration relative to the WT $\left(\mathrm{MIC}_{\text {Resistant strain }}>\mathrm{MIC}_{\mathrm{WT}}\right.$; Fig. 1A). No change in sensitivity was defined when the growth of resistant strain and WT was inhibited at the same drug concentration $\left(\mathrm{MIC}_{\text {Resistant strain }}=\mathrm{MIC}_{\mathrm{WT}}\right.$; Fig. 1B). Collateral sensitivity was defined when the growth of the resistant strain was inhibited at lower concentrations than the WT $\left(\mathrm{MIC}_{\text {Resistant strain }}<\mathrm{MIC}_{\mathrm{WT}}\right.$; Fig. $\left.1 \mathrm{C}\right)$. Collateral sensitivity profiles were used to visualize collateral sensitivity networks with Cytoscape 3.0.1. software.

\section{Isogenic CFP- and YFP-labeled strains}

pZE21 MCS1 (50) plasmid containing kanamycin resistance marker with fluorescently labeled CFP or YFP was electroporated into E. coli MG1655. These labeled isogenic strains were used for the competition assay described below.

\section{Competition assay}

We started this experiment by selecting the resistant mutant toward gentamicin. WT labeled with CFP was gradually adapted to $32 \times \mathrm{MIC}$ gentamicin according to the protocol described above. Then, the CFPlabeled gentamicin-resistant strain was mixed in equal proportions with the WT labeled with YFP. Each strain at about $5 \times 10^{4} \mathrm{CFU} / \mathrm{ml}$ was incubated in $5 \mathrm{ml}$ of LB broth supplemented with various concentrations of cefuroxime $(0,0.5,1,2,4,8$, and $16 \mu \mathrm{g} / \mathrm{ml})$. After 18 hours of incubation at $37^{\circ} \mathrm{C}$ with shaking, serial dilutions were plated onto LB agar plates supplemented with kanamycin $(50 \mu \mathrm{g} / \mathrm{ml})$ and with kanamycin and gentamicin $(8 \mu \mathrm{g} / \mathrm{ml})$. Plate supplemented with kanamycin allowed growth of WT and resistant strain, whereas the growth of WT was inhibited on agar plates supplemented with both kanamycin and gentamicin. Viable cell growth was evaluated at 18 hours after incubation at $37^{\circ} \mathrm{C}$. On the basis of this experiment, we selected the concentration of cefuroxime $(4 \mu \mathrm{g} / \mathrm{ml})$ at which only WT strain survived, whereas gentamicin-resistant strain was eradicated. After this selection step, we continued the exposure of the YFP-labeled WT strain to the increasing concentrations of cefuroxime, as described above. After 7 days of cefuroxime exposure, the strain evolved 128× MIC was selected (YFP-labeled cefuroxime-resistant 
strain). Then, the cefuroxime-resistant strain was mixed in equal proportions with WT cells labeled with CFP. After 18 hours of incubation with gentamicin $(0,0.12,0.25,0.5,1,2$, and $4 \mu \mathrm{g} / \mathrm{ml})$, viable cell growth was determined by plating serial dilutions on agar plates supplemented with kanamycin $(50 \mu \mathrm{g} / \mathrm{ml})$ and kanamycin with gentamicin $(8 \mu \mathrm{g} / \mathrm{ml})$. On the basis of this set of experiments, we were able to select a concentration of gentamicin $(1 \mu \mathrm{g} / \mathrm{ml})$ at which the YFP-labeled cefuroximeresistant strain was killed but the WT strain producing CFP survived.

Next, to exclude any variation in sensitivity between CFP- and YFPlabeled strains and to determine the adaptive repeatability of collateral sensitivity observed for gentamicin- and cefuroxime-resistant strains, we repeated the competition assay by switching the drug toward which labeled strain was adapted (that is, YFP-labeled strain was adapted to gentamicin, and CFP-labeled strain to cefuroxime). The resistant strains were tested for collateral sensitivity according to the above-described method. The results are shown in fig. S2. For completion experiments, strains from overnight cultures were added at 1:1 ratio $\left(5 \times 10^{4}\right)$ to $5 \mathrm{ml}$ of LB broth supplemented with gentamicin $(1 \mu \mathrm{g} / \mathrm{ml})$ or cefuroxime $(4 \mu \mathrm{g} / \mathrm{ml})$ and incubated for 18 hours at $37^{\circ} \mathrm{C}$ with shaking. Strain survival was determined as viable cell count on LB agar supplemented with kanamycin $(50 \mu \mathrm{g} / \mathrm{ml})$ and gentamicin $(8 \mu \mathrm{g} / \mathrm{ml})$ or cefuroxime $(64 \mu \mathrm{g} / \mathrm{ml})$.

\section{Time-kill curves}

About $5 \times 10^{5}$ cells from overnight culture were incubated at $16 \times$ MIC. At different time points during incubation, serial dilutions were plated onto LB agar. Viable cell count was determined after 24 hours of incubation at $37^{\circ} \mathrm{C}$.

\section{MPC determination}

MPC was determined by plating initial inoculums of $10^{9} \mathrm{CFU} / \mathrm{ml}$ from the overnight culture onto LB agar plates supplemented with gentamicin or cefuroxime $\left(1 \times, 2 \times, 4 \times\right.$, and $\left.8 \times \mathrm{MIC}_{\mathrm{WT}}\right)$. Viable cell growth was counted after 24 hours of incubation at $37^{\circ} \mathrm{C}$.

\section{Statistical analysis}

The percent growth inhibition was calculated according to the following formula: $1-\left[A_{600} \mathrm{drug} / A_{600}\right.$ control $]$. Data from MICs are presented as means of triplicate measurements and their SDs. To obtain dose-response growth inhibition curves, we plotted percent inhibition against antibiotic concentrations within twofold dilution. Growth inhibition of the WT and each resistant strain was plotted against drug concentrations with a polynomial interpolation between neighboring data points (that did not affect final data analysis) using R software (http://www.r-project.org). The inhibitory concentration was defined as the lowest drug concentration that prevented $90 \%$ growth $\left(\mathrm{IC}_{90}\right)$. Data derived from competition experiments and time-kill experiments were log-transformed and presented as means and SDs of triplicate measurements.

\section{SUPPLEMENTARY MATERIALS}

www.sciencetranslationalmedicine.org/cgi/content/full/5/204/204ra132/DC1 Table S1. Drug adaptation level and clinical breakpoints.

Table S2. Drug common use.

Table S3. List of possible collateral sensitivity cycles.

Fig. S1. MIC inhibition curves for E. coli MG1655 resistant strains and WT. Fig. S2. Competition assay.

Fig. S3. Collateral sensitivity and collateral resistance distribution.

Fig. S4. MIC inhibition curves for E. coli EC3770 resistant strains and WT.

Fig. S5. MIC inhibition curves for E. coli EC3856 resistant strains and WT.

\section{REFERENCES AND NOTES}

1. A. D. Lopez, C. D. Mathers, M. Ezzati, C. J. L. Murray, D. T. Jamison, Global Burden of Disease and Risk Factors (The World Bank and Oxford Univ. Press, New York, 2006).

2. World Health Organization (WHO), Deaths Estimates for 2008 by Cause for WHO Member States (WHO Health Statistics and Information Systems, Geneva, 2011); http://www.who.int/ healthinfo/global_burden_disease/estimates_country/en/index.html.

3. H. W. Boucher, G. H. Talbot, J. S. Bradley, J. E. Edwards, D. Gilbert, L. B. Rice, M. Scheld, B. Spellberg, J. Bartlett, Bad bugs, no drugs: No ESKAPE! An update from the Infectious Diseases Society of America. Clin. Infect. Dis. 48, 1-12 (2009).

4. D. E. Goldberg, R. F. Siliciano, W. R. Jacobs Jr., Outwitting evolution: Fighting drug-resistant TB, malaria, and HIV. Cell 148, 1271-1283 (2012).

5. A. Pfaller, Antifungal drug resistance: Mechanisms, epidemiology, and consequences for treatment. Am. J. Med. 125, S3-S13 (2012).

6. M. Raviglione, B. Marais, K. Floyd, K. Lönnoroth, H. Getahun, G. B. Migliori, A. D. Harries, P. Nunn, C. Lienhardt, S. Graham, J. Chakaya, K. Weyer, S. Cole, S. H. E. Kaufmann, A. Zumla, Scaling up interventions to achieve global tuberculosis control: Progress and new developments. Lancet 379, 1902-1913 (2012).

7. European Centre for Disease Prevention and Control (ECDC) and European Medicines Agency (EMEA), The Bacterial Challenge: Time to React (Joint Technical Report EMEA/576176) (European Centre for Disease Prevention and Control, Stockholm, 2009); http://www.emea.europa.eu/ docs/en_GB/document_library/Report/2009/11/WC500008770.pdf.

8. P. Borst, Cancer drug pan-resistance: Pumps, cancer stem cells, quiescence, epithelial to mesenchymal transition, blocked cell death pathways, persisters or what? Open Biol. 2, 120066 (2012).

9. K. M. Pluchino, M. D. Hall, A. S. Goldsborough, R. Callaghan, M. M. Gottesman, Collateral sensitivity as a strategy against cancer multidrug resistance. Drug Resist. Updat. 15, 98-105 (2012).

10. M. A. Fischbach, C. T. Walsh, Antibiotics for emerging pathogens. Science 325, 1089-1093 (2009).

11. L. L. Silver, Challenges of antibacterial discovery. Clin. Microbiol. Rev. 24, 71-109 (2011).

12. R. M. Gulick, J. W. Mellors, D. Havlir, J. J. Eron, C. Gonzalez, D. McMahon, D. D. Richman, F. T. Valentine, L. Jonas, A. Meibohm, E. A. Emini, J. A. Chodakewitz, Treatment with indinavir, zidovudine, and lamivudine in adults with human immunodeficiency virus infection and prior antiretroviral therapy. N. Engl. J. Med. 337, 734-739 (1997).

13. F. Clavel, A. J. Hance, HIV drug resistance. N. Engl. J. Med. 350, 1023-1035 (2004).

14. M. A. Fischbach, Combination therapies for combating antimicrobial resistance. Curr. Opin. Microbiol. 14, 519-523 (2011).

15. P. Ball, Conclusions: The future of antimicrobial therapy-Augmentin and beyond. Int. J. Antimicrob. Agents 30 (Suppl. 2), S139-S141 (2007).

16. R. Chait, A. Craney, R. Kishony, Antibiotic interactions that select against resistance. Nature 446, 668-671 (2007).

17. C. A. Ohl, E. S. Dodds Ashley, Antimicrobial stewardship programs in community hospitals: The evidence base and case studies. Clin. Infect. Dis. 1, S23-S28 (2011).

18. C. B. Cunha, C. A. Varughese, E. Mylonakis, Antimicrobial stewardship programs (ASPs): The devil is in the details. Virulence 4, 147-149 (2013).

19. M. S. Niederman, Appropriate use of antimicrobial agents: Challenges and strategies for improvement. Crit. Care Med. 31, 608-616 (2003).

20. R. Masterton, Antibiotic cycling: More than it might seem? J. Antimicrob. Chemother. 55, 1-5 (2005).

21. A. M. Bal, A. Kumar, I. M. Gould, Antibiotic heterogeneity: From concept to practice. Ann. N. Y. Acad. Sci. 1213, 81-91 (2010).

22. D. I. Andersson, D. Hughes, Antibiotic resistance and its cost: Is it possible to reverse resistance? Nat. Rev. Microbiol. 8, 260-271 (2010).

23. P. Komp Lindgren, L. L. Marcusson, D. Sandvang, N. Frimodt-Møller, D. Hughes, Biological cost of single and multiple norfloxacin resistance mutations in Escherichia coli implicated in urinary tract infections. Antimicrob. Agents Chemother. 49, 2343-2351 (2005).

24. L. L. Marcusson, N. Frimodt-Møller, D. Hughes, Interplay in the selection of fluoroquinolone resistance and bacterial fitness. PLoS Pathog. 5, e1000541 (2009).

25. T. Luangtongkum, Z. Shen, V. W. Seng, O. Sahin, B. Jeon, P. Liu, Q. Zhang, Impaired fitness and transmission of macrolide-resistant Campylobacter jejuni in its natural host. Antimicrob. Agents Chemother. 56, 1300-1308 (2012).

26. S. Gagneux, C.D. Long, P. M. Small, T. Van, G. K. Schoolnik, B. J. Bohannan, The competitive cost of antibiotic resistance in Mycobacterium tuberculosis. Science 312, 1944-1946 (2006).

27. A. Rodríguez-Rojas, M. D. Maciá, A. Couce, C. Gómez, A. Castañeda-García, A. Oliver, J. Blázquez, Assessing the emergence of resistance: The absence of biological cost in vivo may compromise fosfomycin treatments for P. aeruginosa infections. PLoS One $\mathbf{5}$, e10193 (2010)

28. J. Björkman, I. Nagaev, O. G. Berg, D. Hughes, D. I. Andersson, Effects of environment on compensatory mutations to ameliorate costs of antibiotic resistance. Science 287, 1479-1482 (2000). 
29. M. Sundqvist, P. Geli, D. I. Andersson, M. Sjölund-Karlsson, A. Runehagen, H. Cars, K. Abelson-Storby, O. Cars, G. Kahlmeter, Little evidence for reversibility of trimethoprim resistance after a drastic reduction in trimethoprim use. J. Antimicrob. Chemother. 65, 350-360 (2010).

30. E. M. Brown, D. Nathwani, Antibiotic cycling or rotation: A systematic review of the evidence of efficacy. J. Antimicrob. Chemother. 55, 6-9 (2005).

31. S. Sarraf-Yazdi, M. Sharpe, K. M. Bennett, T. L. Dotson, D. J. Anderson, S. N. Vaslef, A 9-year retrospective review of antibiotic cycling in a surgical intensive care unit. J. Surg. Res. 176, e73-e78 (2012).

32. D. K. Warren, H. A. Hill, L. R. Merz, M. H. Kollef, M. K. Hayden, V. J. Fraser, S. K. Fridkin, Cycling empirical antimicrobial agents to prevent emergence of antimicrobial-resistant Gram-negative bacteria among intensive care unit patients. Crit. Care Med. 32, 2450-2456 (2004).

33. H. J. van Loon, M. R. Vriens, A. C. Fluit, A. Troelstra, C. van der Werken, J. Verhoef, M. J. Bonten, Antibiotic rotation and development of Gram-negative antibiotic resistance. Am. J. Respir. Crit. Care Med. 171, 480-487 (2005).

34. W. Szybalski, V. Bryson, Genetic studies on microbial cross resistance to toxic agents. I. Cross resistance of Escherichia coli to fifteen antibiotics. J. Bacteriol. 64, 489-499 (1952).

35. C. Tamae, A. Liu, K. Kim, D. Sitz, J. Hong, E. Becket, A. Bui, P. Solaimani, K. P. T ran, H. Yang, J. H. Miller, Determination of antibiotic hypersensitivity among 4,000 single-gene-knockout mutants of Escherichia coli. J. Bacteriol. 190, 5981-5988 (2008).

36. A. Liu, L. Tran, E. Becket, K. Lee, L. Chinn, E. Park, K. Tran, J. H. Miller, Antibiotic sensitivity profiles determined with an Escherichia coli gene knockout collection: Generating an antibiotic bar code. Antimicrob. Agents Chemother. 54, 1393-1403 (2010).

37. W. Szybalski, Genetic studies on microbial cross resistance to toxic agents. IV. Cross resistance of Bacillus megaterium to forty-four antimicrobial drugs. Appl. Microbiol. 2, 57-63 (1954).

38. K. P. Romano, A. Ali, C. Aydin, D. Soumana, A. Ozen, L. M. Deveau, C. Silver, H. Cao, A. Newton, C. J. Petropoulos, W. Huang, C. A. Schiffer, The molecular basis of drug resistance against hepatitis C virus NS3/4A protease inhibitors. PLoS Pathog. 8, e1002832 (2012).

39. M. J. Lee, A. S. Ye, A. K. Gardino, A. M. Heijink, P. K. Sorger, G. MacBeath, M. B. Yaffe, Sequential application of anticancer drugs enhances cell death by rewiring apoptotic signaling networks. Cell 149, 780-794 (2012).

40. R. M. Klevens, J. R. Edwards, C. L. Richards Jr., T. C. Horan, R. P. Gaynes, D. A. Pollock, D. M. Cardo, Estimating health care-associated infections and deaths in U.S. hospitals, 2002. Public Health Rep. 122, 160-166 (2007).

41. B. Allegranzi, S. Bagheri Nejad, C. Combescure, W. Graafmans, H. Attar, L. Donaldson, D. Pittet, Burden of endemic health-care-associated infection in developing countries: Systematic review and meta-analysis. Lancet 377, 228-241 (2011).

42. A. Y. Peleg, D. C. Hooper, Hospital-acquired infections due to Gram-negative bacteria. N. Engl. J. Med. 362, 1804-1813 (2010)

43. K. Drlica, X. Zhao, Mutant selection window hypothesis updated. Clin. Infect. Dis. 44, 681-688 (2007).
44. D. Hughes, D. I. Andersson, Selection of resistance at lethal and non-lethal antibiotic concentrations. Curr. Opin. Microbiol. 15, 555-560 (2012).

45. L. M. Grayson, A. Kucers, S. Crowe, J. McCarthy, J. Mills, J. Mouton, R. Norrby, D. Paterson, M. Pfaller, Kucers' the Use of Antibiotics: A Clinical Review of Antibacterial, Antifungal, Antiparasitic and Antiviral Drugs (Edward Arnold Ltd., London, ed. 6, 2010), vol. 1.

46. P. Imming, C. Sinning, A. Meyer, Drugs, their targets and the nature and number of drug targets. Nat. Rev. Drug Discov. 5, 821-834 (2006).

47. R. L. Gibson, J. L. Burns, B. W. Ramsey, Pathophysiology and management of pulmonary infections in cystic fibrosis. Am. J. Respir. Crit. Care Med. 168, 918-951 (2003).

48. A. G. Bosanquet, P. B. Bell, Novel ex vivo analysis of nonclassical, pleiotropic drug resistance and collateral sensitivity induced by therapy provides a rationale for treatment strategies in chronic lymphocytic leukemia. Blood 87, 1962-1971 (1996).

49. M. Polenakovic, L. Grcevska, S. Dzikova, 20 years after methylprednisolone/chlorambucil treatment in idiopathic membranous nephropathy stage II-III with nephrotic syndrome. Prilozi 27, 5-12 (2006).

50. R. Lutz, H. Bujard, Independent and tight regulation of transcriptional units in Escherichia coli via the $\mathrm{LacR} / \mathrm{O}$, the TetR/O and $\mathrm{AraC} / \mathrm{I}_{1}-\mathrm{I}_{2}$ regulatory elements. Nucleic Acids Res. 25, 1203-1210 (1997).

Acknowledgments: We would like to acknowledge S. Fredholm who began the work on collateral sensitivity in our laboratory. We thank C. Munck and A. Nilsson for discussions regarding collateral sensitivity and help on MIC data analysis and M. Ellabaan for help with determination of collateral sensitivity cycles. We thank K. Schønning for providing us with clinical isolates. We also thank R. Linding and C. Costa for critical reading of this manuscript. Funding: This research was funded by the EU FP7-Health Program Evotar (282004). M.O.A.S. acknowledges additional funding from the Novo Nordisk Foundation, The Lundbeck Foundation, lb Henriksen Foundation, and The Danish Free Research Councils. Author contributions: M.O.A.S. conceived the original idea. L.I. and M.O.A.S. designed the experiments. L.I. performed the experiments. L.I. and M.O.A.S. analyzed the data and wrote the manuscript. Competing interests: M.O.A.S. is cofounder of Microlytic ApS, Biosyntia ApS, and AntibioTx ApS. L.I. declares that she has no competing financial interests.

Submitted 20 May 2013

Accepted 26 August 2013

Published 25 September 2013

10.1126/scitransImed.3006609

Citation: L. Imamovic, M. O. A. Sommer, Use of collateral sensitivity networks to design drug cycling protocols that avoid resistance development. Sci. Transl. Med. 5, 204 ra132 (2013). 